\section{Senioren - Lost in Multimedication}

Ältere Patienten, die dauerhaft fünf oder mehr Medikamente nehmen müssen, verlieren leicht mal den Überblick, wozu die Mittel eigentlich gut sind. Von 754 Hausarztpatienten konnten in einer niederländischen Studie nur 15\% von jedem verordneten Mittel sagen, weshalb sie es einnahmen. Die Patienten waren im Mittel 73 Jahre alt und konnten sich selbst versorgen. Das Verständnis für die Therapie sank mit steigender Zahl der Medikamente und mit zunehmendem Alter. Frauen konnten sich die Indikationen besser merken.

Age and Ageing 2016;45:402-8

\section{Pflege für Babyhaut}

In einer britischen Studie wurde untersucht, wie sich der vierwöchige Einsatz von Oliven- oder Sonnenblumenöl im Vergleich zu keiner Ölanwendung auf die Babyhaut auswirkt. An den Beinen, Armen und dem Rumpf der Babys war die mit Öl gepflegte Haut deutlich besser mit Feuchtigkeit versorgt als die der Vergleichsgruppe. Allerdings zeigte keines der Kinder unabhängig von der Pflege mit Öl stark trockene oder schuppige Haut. Auch der pH-Wert der Babyhaut unterschied sich zwischen den Gruppen nicht. Hingegen unterschiedlich fiel die Untersuchung der Hautstruktur aus. Hier zeigte die oberste Schicht der Epidermis durch den Öleinsatz eine veränderte, weichere Struktur. Aus diesem Grund wollen die Studienautoren eine Hautpflege mit Öl nicht befürworten, da sie nur Empfehlungen aussprechen, wenn sie mehr nützen als schaden. Unabhängig davon wurde aber kein Zusammenhang zwischen Ölanwendungen auf der Babyhaut und der Entstehung von Neurodermitis gefunden.

Cooke A et al. Acta Derm Venereol 2016; 96 : 323-30

\section{Lieber weniger tierisches Eiweiß?}

Proteinbetonte Diäten liegen im Trend doch die Herkunft des Eiweiß ist laut einer US-Studie mit 130.000 Probanden nicht egal. Eine Zunahme der Energiezufuhr aus tierischem Eiweiß um 10\% schlug dabei mit einer um $8 \%$ erhöhten kardiovaskulären Sterblichkeit zu Buche. Dagegen bedeuteten 3\% mehr pflanzliches Eiweiß eine Risikosenkung um $8 \%$.

JAMA Intern Med 2016, online 1. August; doi: 10.1001/jamainternmed.2016.4182

\title{
Bei kritisch kranken Kindern mit der parenteralen Ernährung warten
}

Kinder auf Intensivstationen, die nicht enteral ernährt werden können, bekommen fast regelmäßig schon zu Beginn der Behandlung eine parenterale Ernährung verabreicht. Die Rationale für diese Maßnahme leuchtete ein, allerdings gab es keine Evidenzbasis dafür. Eine internationale Studie liefert nun Daten, die überraschenderweise gegen eine sofortige Ernährung sprechen.

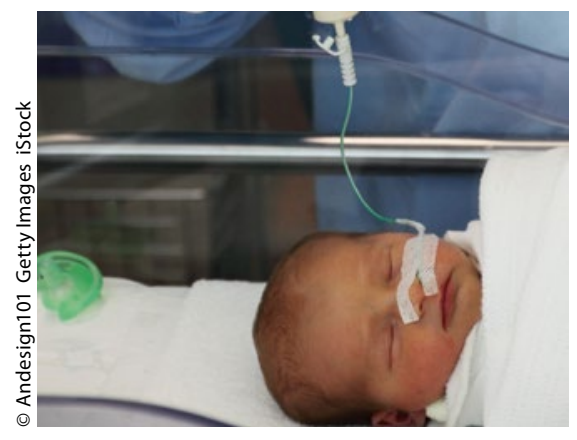

— ine belgische Arbeitsgruppe aus Leu— ven legt nun eine große international angelegte multizentrische, randomisierte, kontrollierte Studie vor, bei der Kliniken aus den Niederlanden und Kanada mitbeteiligt waren. Sie widmet sich der Frage, ob das Vorenthalten parenteraler Ernährung bei kritisch kranken Kindern bis zum Tag 8 vorteilhafter ist im Vergleich zur frühen parenteralen Ernährung. In die Studie wurden alle Altersgruppen vom reifen Neugeborenen bis zum 17-jährigen Jugendlichen aufgenommen. 1.440 Patienten wurden randomisiert: 723 der Patienten erhielten eine frühe parenterale Ernährung innerhalb der ersten 24 Stunden nach der Aufnahme auf der Intensivstation, 717 Patienten erhielten eine parenterale Ernährung erst ab dem Morgen des 8 . Tages der stationären Behandlung.

Beide Gruppen wurden wenn möglich auch enteral ernährt und erhielten intravenöse Mikronutrients (Spurenelemente, Mineralien, Vitamine) ab dem zweiten Tag. Um in beiden Gruppen die gleiche intravenöse Flüssigkeitsmenge zu verabreichen, erhielt die Gruppe der nicht parenteral Ernährten intravenöse Glukose und 0,9\%ige NaCl-Lösung. Die Endpunkte der Studie waren das Neuauftreten von Infektionen während der Behandlung auf der Intensivstation und die Dauer der Intensivbehandlungsbedürftigkeit. Die Mortalität war in beiden
Gruppen gleich hoch. Das Auftreten einer neuen Infektion lag bei den frühzeitig parenteral Ernährten bei $18,5 \%$, bei den spät parenteral Ernährten nur bei $10,7 \%$. In der letztgenannten Gruppe betrug die durchschnittliche Dauer der Intensivbehandlungsbedürftigkeit $\quad 6,5$ Tage, bei den sofort parenteral Ernährten dagegen 9,2 Tage. Auch die Dauer der Beatmungsbedürftigkeit war geringer bei den erst spät parenteral ernährten Kindern. Die Autoren schließen aus ihren Daten, dass es bei kritisch kranken Kindern sinnvoll sei, die parenterale Ernährung erst nach der ersten Woche auf der Intensivstation zu beginnen.

Fivez $T$ et al. Early versus late parenteral nutrition in critically ill children. N Engl J Med 2016; 374:1111-22

\section{Kommentar}

Auf vielen pädiatrischen Intensivstationen wurde und wird die parenterale Ernährung gewissermaßen reflektorisch zum frühestmöglichen Termin begonnen - weil es nahelag, dass dies vernünftig sei. Die Studie von Fivez et al. (genannt PEPaNIC trial) zeigt, wie wichtig es ist, solche Überzeugungen mit stringenten Methoden auf ihre Richtigkeit zu überprüfen. Nilesh Mehta vom Boston Children's Hospital (Critical Care Nutrition) stimmt den Autoren in einem Kommentar zu [N Engl J Med 2016;374: 1190-2]: Zumindest Kinder, die nicht von vornherein stark unterernährt sind, sollten tatsächlich nicht sofort am Aufnahmetag auf einer Intensivstation parenteral ernährt werden.

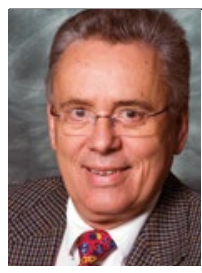

Dr. med. Hartmut Koch

Marienstr. 6

49377 Vechta 